\title{
Modeling and Analytical Simulation of Anterior Polymerization in the Presence of an Inert Material
}

\section{*11JIMADA, AM; OLAYIWOLA, RO; M. D. SHEHU; COLE, AT; A. A. MOHAMMED}

\author{
Department of Mathematics, \\ Federal University of Technology, Minna, Nigeria. \\ E-mail.: olaviwola.rasaq@futminna.edu.ng
}

\begin{abstract}
The ability to fabricate advanced materials with specific properties efficiently requires a complete understanding of the polymerization kinetics and the effect of several preparative variables such as temperature, monomer and initiator. This paper presents an analytical method for describing anterior polymerization in two adjacent thin layers. Both the initial temperatures and initial monomer and initiator concentrations are assumed to depend on the space variable. We prove the existence and uniqueness of solution of the model by actual solution method. The equations are solved using parameter-expanding method and eigenfunctions expansion technique. The results obtained were discussed. The study shows that the Frank-Kamenetskii number and frequency factors of the two reactions have significant effects on the propagation of the polymerization wave. $\odot$ JASEM

http://dx.doi.org/10.4314/jasem.v21i1.11
\end{abstract}

Keywords: Polymerization, anterior polymerization, polymers, Arrhenius kinetics, parameter-expanding method, eigenfunctions expansion technique.

Polymers, either synthetic or natural, are present in every aspect of our daily lives. Many modern functional materials, pharmaceutical equipment, electronic devices, automobile parts, etc., have polymeric components. Polymers are replacing traditional materials because of their low cost and special applications. Our lives have been thoroughly changed with the advent of mobile phones, computers, refrigerators, electrical domestic appliances, television, etc.; all of these appliances have parts made of synthetic polymeric materials to a large extent. Polymeric materials are also everywhere in our homes: floor carpeting, glue, pipes, paint, wallpaper, foils, electric insulation and mouldings are examples of components based on synthetic polymers (Olayiwola et al., 2013).

A promising new technique for synthesizing uniform polymers and polymeric networks in a rapid fashion is frontal polymerization (FP). Frontal polymerization (FP) is a chemical process whereby monomer is converted to polymer via a localized reaction zone (Washington and Steinbock, 2003).

The development of new polymers and the modification and enhancement of the old ones are goals of many researchers in both industry and academia. Almeida et al. (2008) developed a mathematical model for the free radical polymerization of styrene. Cardarelli et al. (2005) used numerical simulation to study the influence of reaction kinetics on one-step frontal polymerization in one dimension in the absence of material diffusion. They neglected the material diffusion and showed that the long-time behaviour of systems governed by approximate kinetics significantly differs from the long-time behaviour of systems governed by Arrhenius kinetics. Olayiwola et al. (2013) presented a mathematical model for the free radical Polymerization in the presence of material diffusion. They assume both the initial monomer concentration and initial temperature of the mixture depend on space variable. They discovered that the mixture temperature and monomer concentration are significantly influenced by the Frank-Kamenetskii number, material diffusion coefficient and thermal diffusivity of the mixture. Comissiong et al. (2006) considered the steady propagation of a onedimensional frontal polymerization (FP) wave in a sandwich-type two-layer model. A single stationary solution is found for the reactive layer in the presence of very thin inert layers. They carry out a linear stability analysis of the uniformly propagating waves.

The objective of this paper is to obtain an analytical solution for describing polymerization in the presence of an inert material.

\section{MATERIALS AND METHODS}

We consider two adjacent thin layers in formulating a mathematical model for the problem. One layer is made up of an inert material, and the other layer is a reactive mixture, which initially consists of monomer and initiator. There is thermal contact between the two layers. The polymerization process occurring in the reactive layer is exothermic, and therefore there is exchange of heat between the layers as shown in fig. 1. 


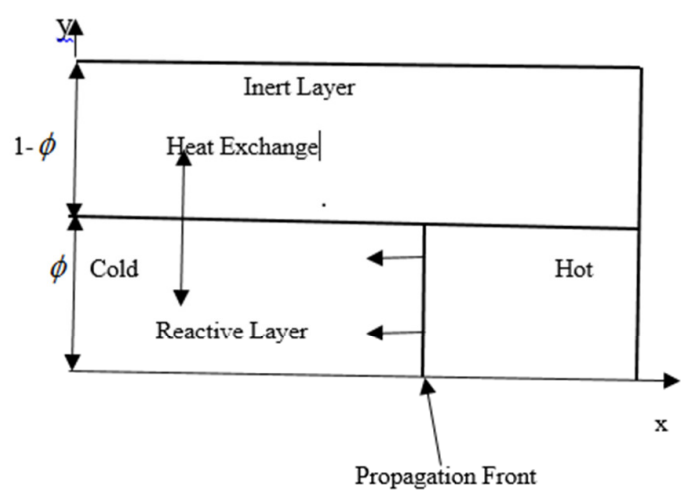

Fig. 1: Diagram showing system under study.
The polymerization process occurring in the reactive layer is the free-radical polymerization which involves a standard sequence of chemical reactions (Odian, 1991). The process begins when the initiator decomposes, forming two radicals. Each radical can then combine with a monomer, initiating a polymer chain. A polymer chain grows by combining with another monomer to form a longer chain, and terminates by combining with a radical, either another growing chain or an initiator radical. Thus, the kinetic scheme involves the decomposition step, initiation step, propagation step and the termination step. This kinetic scheme can be represented by the Kinetic equations for the concentration of the initiator and the monomer.

\section{For initiator:}

$$
\frac{\partial I}{\partial t}+k_{d}^{0} I e^{-\frac{E_{d}}{R T_{r}}}=0
$$

For monomer:

$$
\frac{\partial M}{\partial t}+k_{e}^{0} M \sqrt{I} e^{-\frac{E_{e}}{R T_{r}}}=0
$$

\section{The heat equation in the reactive layer has the form}

$$
\phi c_{r} \rho_{r} \frac{\partial T_{r}}{\partial t}=\phi \lambda_{r} \frac{\partial^{2} T_{r}}{\partial x^{2}}-\alpha\left(T_{r}-T_{i}\right)+\phi \Delta h k_{e}^{0} M \sqrt{I} e^{-\frac{E e}{R T_{r}}}
$$

The heat balance in the inert layer is given by the equation

$$
(1-\phi) c_{i} \rho_{i} \frac{\partial T_{i}}{\partial t}=(1-\phi) \lambda_{i} \frac{\partial^{2} T_{i}}{\partial x^{2}}-\alpha\left(T_{i}-T_{r}\right)
$$

Here, we assume both the initial concentration of monomer and initiator and initial temperature of the mixture depend on the space variable $x$ and we impose the adiabatic boundary conditions on the temperatures. Thus, the initial and boundary conditions were formulated as follows:

$$
\left.\begin{array}{c}
I(x, 0)=I_{0}\left(1-\frac{x}{L}\right) \\
M(x, 0)=M_{0}\left(1-\frac{x}{L}\right) \\
T_{r}(x, 0)=\in T_{0}\left(1-\frac{x}{L}\right)+T_{0}, \quad T_{r x}(0, t)=T_{0}, \quad T_{r x}(L, t)=T_{0} \\
T_{i}(x, 0)=\in T_{0}\left(1-\frac{x}{L}\right)+T_{0}, \quad T_{i x}(0, t)=T_{0}, \quad T_{i x}(L, t)=T_{0}
\end{array}\right\},
$$

where $I$ is the concentration of the initiator, $M$ is the monomer concentration, $t$ is the time, $k_{d}$ and $k_{e}$ are the decomposition and the polymerization reaction rate parameters which depend on the temperature $T_{r}$ of the reactive layer, $R$ is the gas constant, $k_{d}^{0}, k_{e}^{0}$ are the frequency factors of the two reactions, $E_{d}, E_{e}$ are the 
activation energies of the two reactions, $\lambda_{r}$ is the thermal conductivity of the reactive layer, $\lambda_{i}$ is the thermal conductivity of the inert layer, $\Delta h$ is the enthalpy of the reaction, $c_{r}$ is the specific heat capacity of the reactive layer, $c_{i}$ is the specific heat capacity of the inert layer, $\rho$ is the mixture density, $T_{i}$ is the temperature of the inert layer, $T_{r}$ is the temperature of the reactive layer, $\phi$ is the ratio of the thickness of the reactive layer to the total thickness of the two layers.

\section{Method of Solution: Non-dimensionalization}

Here, we non-dimensionalized equations (1) - (5), using the following dimensionless variables:

$$
\left.\begin{array}{ccc}
t^{\prime}=\frac{t}{t_{0}}, \quad x^{\prime}=\frac{x}{L}, & I^{\prime}=\frac{I}{I_{0}}, \quad M^{\prime}=\frac{M}{M_{0}}, & \gamma=\frac{E_{d}}{E_{e}}, \\
\theta=\frac{\left(T_{r}-T_{0}\right)}{\in T_{0}}, & \varphi=\frac{\left(T_{i}-T_{0}\right)}{\in T_{0}}, & \in=\frac{R T_{0}}{E_{e}}
\end{array}\right\},
$$

where $t_{0}$ is the initial time for ignition to occur and we obtain

$$
\begin{aligned}
& \frac{\partial I}{\partial t}=-\beta I e^{\frac{\gamma \theta}{1+\epsilon \theta}} \\
& \frac{\partial M}{\partial t}=-\sigma M \sqrt{I} e^{\frac{\theta}{1+\epsilon \theta}} \\
& \frac{\partial \theta}{\partial t}=\lambda_{1} \frac{\partial^{2} \theta}{\partial x^{2}}-\alpha_{1}(\theta-\varphi)+\delta M \sqrt{I} e^{\frac{\theta}{1+\epsilon \theta}} \\
& \frac{\partial \varphi}{\partial t}=\lambda_{2} \frac{\partial^{2} \varphi}{\partial x^{2}}-\alpha_{2}(\varphi-\theta) .
\end{aligned}
$$

Together with initial and boundary conditions:

$$
\begin{aligned}
& I(x, 0)=(1-x) \\
& M(x, 0)=(1-x) \\
& \left.\theta(x, 0)=(1-x), \quad \theta_{x}(0, t)=0, \quad \theta_{x}(1, t)=0\right\}, \\
& \varphi(x, 0)=(1-x), \quad \varphi_{x}(0, t)=0, \quad \varphi_{x}(1, t)=0
\end{aligned}
$$

where $\lambda_{1}=\frac{t_{0}}{c_{r} \rho_{r} L^{2}} \lambda_{r}=$ Reactive layer scaled thermal conductivity, $\lambda_{2}=\frac{t_{0}}{\rho_{i} c_{i} L^{2}} \lambda_{i}=$ Inert layer scaled thermal conductivity, $\quad \alpha_{1}=\frac{\alpha t_{0}}{\phi c_{r} \rho_{r}}, \quad \alpha_{2}=\frac{\alpha t_{0}}{(1-\phi) \rho_{i} c_{i}}, \quad \delta=\frac{\Delta h K_{e}^{0} M_{0} t_{0} \sqrt{I_{0}} e^{-\frac{E_{e}}{R T_{0}}}}{c_{r} \rho_{r} \in T_{0}}=$ FrankKamenetskii parameter, $\quad \beta=K_{d}^{0} t_{0} e^{-\frac{E_{d}}{R T_{0}}}, \quad \sigma=K_{e}^{0} \sqrt{I_{0}} t_{0} e^{-\frac{E_{e}}{R T_{0}}}$

\section{Existence and Uniqueness of Solution}

Theorem 1: Let $\alpha_{2}=\alpha_{1}=\alpha, \sigma=\delta$. Then the equations (7) - (10) with initial and boundary conditions (11) has a unique solution for all $t \geq 0$. 
Proof: Let $\alpha_{2}=\alpha_{1}=\alpha, \sigma=\delta$ and $\psi(x, t)=\theta(x, t)+M(x, t)+\varphi(x, t)$, we obtain

$$
\begin{array}{ll}
\frac{\partial I}{\partial t}=-\beta I \exp \left(\frac{\gamma \theta}{1+\in \theta}\right), \quad I(x, 0)=1-x & \\
\frac{\partial \psi}{\partial t}=\lambda_{1} \frac{\partial^{2} \theta}{\partial x^{2}}+\lambda_{2} \frac{\partial^{2} \varphi}{\partial x^{2}}, & \psi(x, 0)=3(1-x), \quad \theta_{x}(0, t)=0, \quad \theta_{x}(1, t)=0, \\
\theta_{x}(0, t)=0, \quad \theta_{x}(1, t)=0
\end{array}
$$

Integrating both sides with respect to $x$, we obtain the solution of problem (13) as

$\psi(x, t)=\theta(x, t)+M(x, t)+\varphi(x, t)=\pi=$ Constant

Then, we obtain

$$
M(x, t)=\psi(x, t)-(\theta(x, t)+\varphi(x, t))
$$

$\varphi(x, t)=\psi(x, t)-(\theta(x, t)+M(x, t))$

$(16)$

$\theta(x, t)=\psi(x, t)-(\varphi(x, t)+M(x, t))$

(17)

By direct integration, we obtain the solution of (12) as

$$
I(x, t)=\exp \left(-\beta \int \exp \left(\frac{\gamma \theta}{1+\in \theta}\right) . \partial t+C\right)
$$

Hence, there exists a unique solution of problem (7) - (10). This completes the proof.

\section{Analytical Solution}

Here, we let $\gamma=1$ and solve equations (7) - (11) using parameter-expanding method (where details can be found in $\mathrm{He}, 2006$ ) and eigenfunctions expansion method (where details can be found in Myint-U and Debnanth, 1987).

Ayeni (1982) has shown that $\exp \left(\frac{\theta}{1+\in \theta}\right)$ can be approximated as $1+(e-2) \theta+\theta^{2}$. In our analysis we are going to take an approximation of the form:

$$
\exp \left(\frac{\theta}{1+\in \theta}\right) \approx 1+(e-2) \theta
$$

So that equations (7) - (10) can be approximated as:

$$
\begin{aligned}
& \frac{\partial I}{\partial t}=-\beta I(1+(e-2) \theta) \\
& \frac{\partial M}{\partial t}=-\sigma M \sqrt{I}(1+(e-2) \theta) \\
& \frac{\partial \theta}{\partial t}=\lambda_{1} \frac{\partial^{2} \theta}{\partial x^{2}}-\alpha_{1}(\theta-\phi)+\delta M \sqrt{I}(1+(e-2) \theta) \\
& \frac{\partial \phi}{\partial t}=\lambda_{2} \frac{\partial^{2} \phi}{\partial x^{2}}-\alpha_{2}(\phi-\theta)
\end{aligned}
$$

We let

$$
\alpha_{1}=h \in, \quad \sigma=p \in, \quad \delta=q \in, \quad \beta=r \in
$$




$$
\left.\begin{array}{l}
\theta(x, t)=\theta_{0}(x, t)+\in \theta_{1}(x, t)+\ldots \\
\phi(x, t)=\phi_{0}(x, t)+\in \phi_{1}(x, t)+\ldots \\
I(x, t)=I_{0}(x, t)+\in I_{1}(x, t)+\ldots \\
M(x, t)=M_{0}(x, t)+\in M_{1}(x, t)+\ldots
\end{array}\right\}
$$

Substituting (24) into (20) - (23) and processing, we obtain

$$
\begin{aligned}
& \frac{\partial \theta_{0}}{\partial t}=\lambda_{1} \frac{\partial^{2} \theta_{0}}{\partial x^{2}}, \quad \theta_{0}(x, 0)=1-x, \quad \theta_{0 x}(0, t)=0, \quad \theta_{0 x}(1, t)=0 \\
& \frac{\partial \phi_{0}}{\partial t}=\lambda_{2} \frac{\partial^{2} \phi_{0}}{\partial x^{2}}-\alpha_{2}\left(\phi_{0}-\theta_{0}\right), \quad \phi_{0}(x, 0)=1-x, \quad \phi_{0 x}(0, t)=0, \quad \phi_{0 x}(1, t)=0 \\
& \frac{\partial I_{0}}{\partial t}=0, \quad I_{0}(x, 0)=1-x \\
& \frac{\partial M_{0}}{\partial t}=0, \quad M_{0}(x, 0)=1-x \\
& \frac{\partial \theta_{1}}{\partial t}=\lambda_{1} \frac{\partial^{2} \theta_{1}}{\partial x^{2}}-h\left(\theta_{0}-\phi_{0}\right)-q M_{0} \sqrt{I_{0}}\left(1+(e-2) \theta_{0}\right), \theta_{1}(x, 0)=0, \theta_{1 x}(0, t)=0, \theta_{1 x}(1, t)=0 \\
& \frac{\partial \phi_{1}}{\partial t}=\lambda_{2} \frac{\partial^{2} \phi_{1}}{\partial x^{2}}+p x(1-x) \frac{\partial \phi_{0}}{\partial x}-\alpha_{2}\left(\phi_{1}-\theta_{1}\right), \quad \varphi_{1}(x, 0)=0, \quad \phi_{1 x}(0, t)=0, \quad \phi_{1 x}(1, t)=0 \\
& \frac{\partial I_{1}}{\partial t}=-r I_{0}\left(1+(e-2) \theta_{0}\right), \quad I_{1}(x, 0)=0 \\
& \frac{\partial M_{1}}{\partial t}=-p M_{0} \sqrt{I_{0}}\left(1+(e-2) \theta_{0}\right), \quad M_{1}(x, 0)=0
\end{aligned}
$$

Using eigenfunctions expansion method and direct integration, we obtain the solution of equations (25) - (32) as

$$
\begin{aligned}
& \theta_{0}(x, t)=1+\sum_{n=1}^{\infty} A e^{-c t} \cos n \pi x \\
& \phi_{0}(x, t)=\left(2-e^{-\alpha_{2} t}\right)+\sum_{n=1}^{\infty}\left(A e^{-d t}-\alpha_{2} \sum_{n=1}^{\infty} D\left(e^{-c t}-e^{-d t}\right)\right) \cos n \pi x \\
& I_{0}(x, t)=(1-x) \\
& M_{0}(x, t)=(1-x) \\
& \theta_{1}(x, t)=\theta_{10}(t)+\sum_{n=1}^{\infty} \theta_{1 n}(t) \cos n \pi x \\
& \phi_{1}(x, t)=\phi_{10}(t)+\sum_{n=1}^{\infty} \phi_{1 n}(t) \cos n \pi x
\end{aligned}
$$


Modeling and Analytical Simulation of Anterior Polymerization

106

$$
\begin{aligned}
& I_{1}(x, t)=-r(1-x)(e-2) \sum_{n=1}^{\infty} B \cos n \pi x-r(1-x)\left(t+(e-2)\left(t-\sum_{n=1}^{\infty} B e^{-c t} \cos n \pi x\right)\right) \\
& M_{1}(x, t)=-p(1-x)^{\frac{3}{2}}(e-2) \sum_{n=1}^{\infty} B \cos n \pi x-p(1-x)^{\frac{3}{2}}\left(t+(e-2)\left(t-\sum_{n=1}^{\infty} B e^{-c t} \cos n \pi x\right)\right)
\end{aligned}
$$

where

$$
\begin{aligned}
& \theta_{10 .}(t)=R t+\frac{2 h}{\alpha_{2}}\left(e^{-\alpha_{2} t}-1\right) \\
& \theta_{1 n}(t)=\frac{3 q(e-2) E}{2 c}\left(1-e^{-c t}\right)+\frac{1}{80} q(e-2) \sum_{n=1}^{\infty} A F t e^{-c t}+ \\
& h \sum_{n=1}^{\infty}\left(\frac{A}{c-d}\left(e^{-d t}-e^{-c t}\right)-\alpha_{2} \sum_{n=1}^{\infty} D\left(t e^{-c t}-\frac{1}{c-d}\left(e^{-d t}-e^{-c t}\right)\right)\right)-h \sum_{n=1}^{\infty} A t e^{-c t} \\
& \phi_{10 .}(t)=2 \alpha_{2}\left(R\left(\frac{1}{\alpha_{2}} t-\frac{1}{\alpha_{2}}\left(1-e^{-\alpha_{2} t}\right)\right)+\frac{2 h}{\alpha_{2}}\left(t e^{-\alpha_{2} t}-\frac{1}{\alpha_{2}}\left(1-e^{-\alpha_{2} t}\right)\right)\right) \\
& \phi_{1 n}(t)=\alpha_{2} \sum_{n=1}^{\infty}\left(\begin{array}{c}
\frac{1}{80} q(e-2) \sum_{n=1}^{\infty} A F\left(\frac{1}{d-c} t e^{-c t}-\frac{1}{(d-c)^{2}}\left(e^{-c t}-e^{-d t}\right)\right. \\
\frac{3 q(e-1)}{2 c}\left(\frac{1}{d}\left(1-e^{-d t}\right)-\frac{1}{d-c}\left(e^{-c t}-e^{-d t}\right)\right)+ \\
\frac{A}{c-d}\left(t e^{-d t}-\frac{1}{(d-c)}\left(e^{-c t}-e^{-d t}\right)\right)- \\
h \sum_{n=1}^{\infty}\left(\begin{array}{c}
\alpha_{2} \sum_{n=1}^{\infty} D\left(\frac{1}{d-c} t e^{-c t}-\frac{1}{(d-c)^{2}}\left(e^{-c t}-e^{-d t}\right)\right) \\
h \sum_{n=1}^{\infty} A\left(\frac { 1 } { c - d } \left(t e^{-d t}-\frac{1}{(d-c)}\left(e^{-c t}-e^{-d t}\right)\right.\right.
\end{array}\right)
\end{array}\right)-
\end{aligned}
$$

JUMADA, AM; OLAYIWOLA, RS; M. D. SHEHU; COLE, AT; A. A. MOHAMMED 


$$
\begin{aligned}
& A=\frac{2\left(1-(-1)^{n}\right)}{n^{2} \pi^{2}}, \quad B=\frac{A}{c}, \quad d=\alpha_{2}+\lambda_{2} n^{2} \pi^{2}, \quad c=\lambda_{1} n^{2} \pi^{2}, \quad D=\frac{A}{d+c}, \\
& E=\frac{2 \sqrt{n}-\sqrt{2}(-1)^{n} \text { FresnelC }(\sqrt{2 n})}{n^{\frac{5}{2}} \pi^{2}}, \quad R:=\frac{4}{5} q(e-1)+\frac{3}{2} q(e-1) \sum_{n=1}^{\infty} A E+2 h \\
& F=\frac{30 \sqrt{n}-30(-1)^{2 n} \text { FresnelC }(2 \sqrt{n})+15 \text { FresnelC }(2 \sqrt{n})+32 n^{\frac{5}{2}} \pi^{2}}{n^{\frac{5}{2}} \pi^{2}}
\end{aligned}
$$

Note that

$$
\text { FresnelC }(x)=\int_{0}^{x} \cos \left(\frac{1}{2} \pi t^{2}\right) d t
$$

The computations were done using computer symbolic algebraic package MAPLE.

\section{RESULTS AND DISCUSSION}

We solve the systems of coupled nonlinear partial differential equations describing polymerization in the presence of an inert material analytically. We decouple the equations using parameter-expanding method and solve the resulting equations using Eigen functions expansion technique. Analytical solutions of equations (7) (11) are computed for the following parameter values:

$$
\delta=0.4, \quad \lambda_{1}=0.1, \quad \lambda_{2}=0.1, \quad \alpha_{1}=0.00025, \quad \in=0.01, \quad \sigma=0.2, \quad \beta=0.2, \quad \alpha_{2}=0.125 \text {. }
$$

The following figures explain the temperatures, initiator mass fraction and monomer mass fraction distributions against different dimensionless parameters.

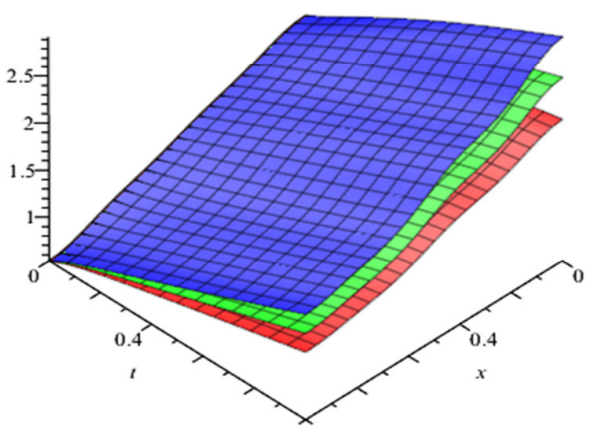

Fig. 2: Variation of reactive layer temperature $\theta(x, t)$ with Frank-Kamenetskii number $\delta$. 


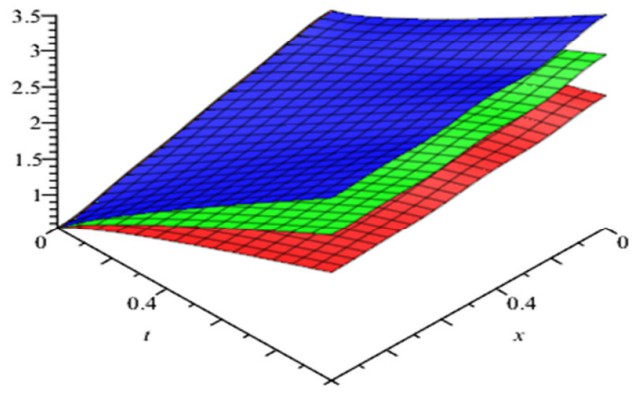

Fig. 3: Variation of inert layer temperature $\varphi(x, t)$ with Frank-Kamenetskii number $\delta$.

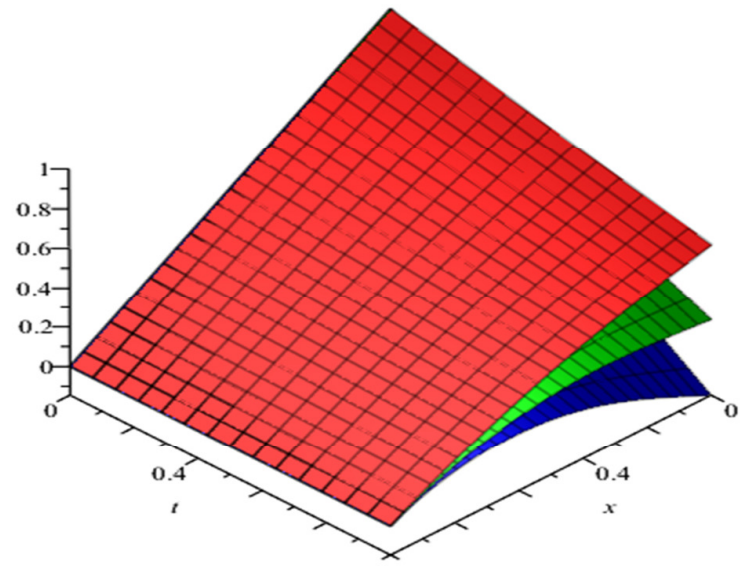

Fig. 4: Variation of monomer concentration $M(x, t)$ with frequency factor for polymerization reaction $\sigma$.

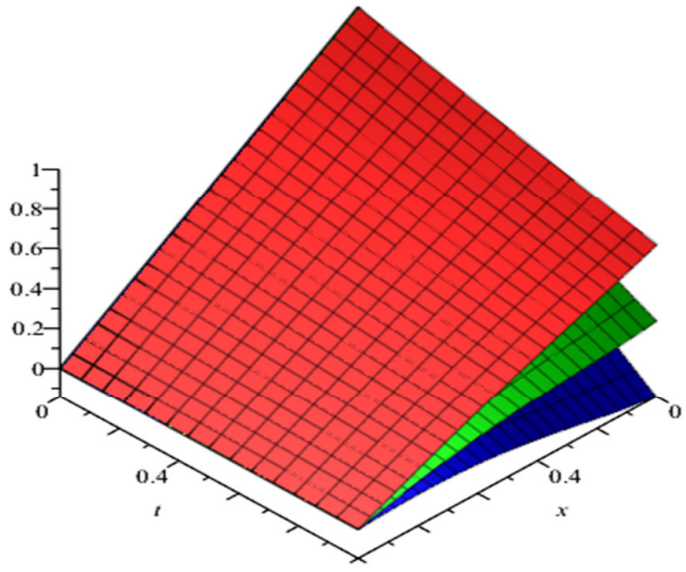

Fig. 5: Variation of initiator concentration $I(x, t)$ with frequency factor for decomposition reaction $\beta$.

Fig. 2 shows the effect of Frank-Kamenetskii number $(\delta)$ on the reactive layer temperature profile. It is observed that the reactive layer temperature increases significantly with time and decreases with distance. Clearly, the Frank-Kamenetskii number enhances the reactive layer temperature. This is as a result of increase in heat of reaction because the reaction that occurred in this layer is exothermic.

Fig. 3 depicts the effect of Frank-Kamenetskii number $(\delta)$ on the inert layer temperature profile. It is observed that the inert layer temperature increases significantly with time and decreases with distance. Clearly, the Frank-Kamenetskii number enhances the 
inert layer temperature. This is as a result of exchange of heat between the layers.

Fig. 4 presents the effect of frequency factor for polymerization reaction $(\sigma)$ on the monomer concentration profile. It is observed that the monomer concentration decreases significantly with time and distance. Clearly, the frequency factor for polymerization reaction decreases the monomer concentration.

Fig. 5 displays the effect of frequency factor for decomposition reaction $(\beta)$ on the initiator concentration profile. It is observed that the initiator concentration decreases significantly with time and distance. Clearly, the frequency factor for decomposition reaction decreases the initiator concentration.

These curves are in semi quantitative agreement with experiment.

Note that the effects observed in figs. 4 and 5 are of great economic importance, since the major reason for using Frontal Polymerization for polymer synthesis is conversion. For polymer synthesis to be effective there must be rapid conversion without the use of solvent and there mustn't be initiator 'burn out', that is, a situation when all the initiator has decomposed before the monomer has been completely reacted. However, if conversion is low and the product must be purified, those advantages will be non-existent.

Conclusion: We have formulated and solved analytically a mathematical model of polymerization in the presence of an inert material to determine the concentration and temperature distributions. In particular, we have proved by actual solution method that the model formulated has a unique solution for all $t \geq 0$. We decoupled the equations using parameter expanding method and solved the resulting equations using Eigen functions expansion technique. Finally, we have provided the graphical summaries of the system responses.

\section{REFERENCES}

Almeida, AS; Wada, K; Secchi, AR (2008). Simulation of Styrene Polymerization Reactors: Kinetics and Thermodynamic Modeling. Brazilian Journal of Chemical Engineering. 25(2): 337-349.

Ayeni, RO (1982). On the explosion of Chainthermal Reactions. J. Austral. Math. Soc. (Series B). 24: 194-202.

Cardarelli, SA; Golovaty, D; Gross, LK; Gyrya, VT; Zhu, J (2005). A Numerical Study of One-Step Models of Polymerization: Frontal versus Bulk Mode. Elsevier Physica D. 206:145-165.

Comissiong, DMG; Gross, LK; Volpert, VA (2006). Frontal polymerization in the presence of an inert material. Journal of Engineering Mathematics. 54: 389-402.

He, JH (2006): Some asymptotic methods for strongly nonlinear equations, Int. J. Modern Phys. B. 20(10): 1141 - 1199.

Myint-U, T; Debnath, L (1987). Partial Differential Equation for Scientists and Engineers. PTR Prentice - Hall, Englewood Cliffs, New Jersey 07632.

Odian, GG (1991). Principles of Polymerization, 3rd edition. New York, Wiley-Interscience, 768.

Olayiwola, RO; Durojaye, MO; Immam, MS; Shuaib, SE (2013). A Mathematical Study of One-Step Models of Polymerization. Pacific Journal of Science and Technology. 14(2):153- 162. http://www.akamaiuniversity.us/PJST.htm

Washington, RP; Steinbock, O (2003). Frontal FreeRadical Polymerization: Applications to Materials Synthesis. Polymer News, 28: 303-310 